\title{
On sharply 2-transitive groups with point stabilizer of exponent $2^{n} \cdot 3$
}

\section{Enrico Jabara}

To cite this article: Enrico Jabara (2018) On sharply 2-transitive groups with point stabilizer of exponent $2^{n} \cdot 3$, Communications in Algebra, 46:2, 544-551, DOI: 10.1080/00927872.2017.1324864

To link to this article: https://doi.org/10.1080/00927872.2017.1324864

Accepted author version posted online: 08

May 2017.

Published online: 08 May 2017.

Submit your article to this journal $₫$

Џ Article views: 39

Q View related articles $\sqsubset$

View Crossmark data \lceil 


\title{
On sharply 2-transitive groups with point stabilizer of exponent $2^{n} \cdot 3$
}

\author{
Enrico Jabara \\ Dipartimento di Filosofia e Beni Culturali, Università di Ca' Foscari, Venezia, Italy
}

\begin{abstract}
We describe sharply 2-transitive groups whose point stabilizer is a nilpotent $\{2,3\}$-group without elements of order 9 and, more generally, in which the third power of each element belongs to the FC-center. In particular, we will prove that these groups are finite.
\end{abstract}

\author{
ARTICLE HISTORY \\ Received 26 January 2016 \\ Communicated by S. Bazzoni

\section{KEYWORDS} \\ FC-group; Frobenius group; \\ near-domain; near-field; \\ sharply 2-transitive \\ permutation group
}

2000 MATHEMATICS SUBJECT CLASSIFICATION 20B22; 20F24; 20F28; 16 Y30

\section{Introduction}

Let $G$ be a sharply 2-transitive permutation group acting on a set $\Omega$ (finite or infinite, with $|\Omega| \geq 2$ ), that is, $G$ is transitive on $\Omega$ and only the identity of $G$ fixes more than one element of $\Omega$.

In the finite case, sharply 2-transitive groups have been classified by Zassenhaus [15], in particular, they are split, that is, they have always a normal abelian subgroup $N$ which is regular on $\Omega$.

In the infinite case the situation is more complex and recently examples were built of non-split sharply 2-transitive groups [11].

In some cases, imposing special conditions on the structure of a point stabilizer $G_{\alpha}=\{g \in G$ । $g(\alpha)=\alpha\}$ of $G(\alpha \in \Omega)$, it can be shown that $G$ is split. This is the case in which every conjugacy class of $G_{\alpha}$ is finite ([6], Theorem 9.6), $G_{\alpha}$ is a 2-group [12] and $G_{\alpha}$ has exponent 3 or 6 [7]. In this note we generalize Mayr's result proving the following

Theorem 1.1. Let $G$ be a sharply 2-transitive permutation group on a set $\Omega$, and let $H=G_{\alpha}$ be the stabilizer of an element $\alpha \in \Omega$. If $H$ is nilpotent and has exponent $2^{n} \cdot 3$ with $n \geq 1$, then $G$ is finite.

If $X$ is a group and $g \in X$, by $g^{X}=\left\{g^{x} \mid x \in X\right\}$, we denote the conjugacy class of $g$ in $X$. We define the FC-center of $X$ as the set

$$
\widehat{Z}(X)=\left\{g \in X|| g^{X} \mid<\infty\right\},
$$

which one can easily prove to be a characteristic subgroup of $X$. A group $X$ is said to be a FC-group if $X=\widehat{Z}(X)$.

Theorem 1.1 is consequence of a more general result.

Theorem 1.2. Let $G$ be a sharply 2-transitive permutation group on a set $\Omega$, and let $H=G_{\alpha}$ be the stabilizer of an element $\alpha \in \Omega$. If $H$ is a $\{2,3\}$-group and $H / \widehat{Z}(X)$ has exponent dividing 3 , then $|\Omega| \in$ $\left\{5^{2}, 7^{2}, 17^{2}\right\}$ or $\Omega$ has prime order, in particular, $G$ is finite. 


\section{Notation and Preliminary Results}

In the following, $G$ denotes a sharply 2 -transitive permutation group on a set $\Omega, \alpha$ a fixed element of $\Omega$ and $H=G_{\alpha}$ the stabilizer in $G$ of $\alpha$. An element $g \in G$ is called regular if $g$ displaces all elements of $\Omega$ or, equivalently,

$$
g \in G \backslash \bigcup_{x \in G} H^{x}=G \backslash \bigcup_{\omega \in \Omega} G_{\omega}
$$

Clearly $H$ is malnormal in $G$, that is, $H \cap H^{g}=1$ for every $g \in G \backslash H$.

Theorem 2.1 ([3], Theorem 20.7.1). Let $\omega_{1}, \omega_{2} \in \Omega$ and suppose that at most one element taking $\omega_{1}$ in $\omega_{2}$ is regular. Then the identity and the regular elements of $G$ form a transitive normal abelian subgroup $N$.

Lemma 2.2 ([3], Lemma 20.7.1). There exists one and only one involution in $G$ which interchanges a specified pair of distinct elements $\omega_{1}, \omega_{2} \in \Omega$.

Lemma 2.3 ([3], Lemmas 20.7.2 and 20.7.4). The involutions of $G$ are in a single conjugacy class. The product of two different involutions is a regular element of $G$.

Lemma 2.4 ([3], Lemma 20.7.3 and Theorem 12.5.2). If the involutions of $G$ are not regular, then in $H$ there is a unique involution, which belongs to the center of $H$. In particular, a 2-subgroup of finite exponent of $H$ is cyclic or quaternion, and hence finite.

Let $J$ be the set of involutions of $G$ and put $J^{2}=\{j k \mid j, k \in J\}$. If $X$ is a subset of $G$, we define $X^{\#}=X \backslash\{1\}$.

Lemma 2.5 ([6], II.4.1.b and II.9.2). If the involutions of $G$ are not regular, then $\left(J^{2}\right)^{\#}$ is a conjugacy class in G. Moreover, every element of $\left(J^{2}\right)^{\#}$ has prime order $p \neq 2$ or infinite order.

The following is a standard definition.

Definition I. Let $G$ be a sharply 2-transitive permutation group.

If an involution (and hence any involution) of $G$ is not regular, we define $\operatorname{char}(G)$, the characteristic of $G$, to be $p$ if an element of $\left(J^{2}\right)^{\#}$ has order $p$ and $\operatorname{char}(G)=0$ if an element of $\left(J^{2}\right)^{\#}$ has infinite order.

If the involutions of $G$ are regular, we define $\operatorname{char}(G)=2$.

Lemma 2.6 ([6], II.9.2). If $\operatorname{char}(G)=p>0$, then $H$ contains a cyclic subgroup of order $p-1$.

Remark A. If $\operatorname{char}(G)=0$, then we can prove that $H$ contains elements of infinite order. Since we will consider only the case in which $H$ is periodic and contains elements of even order, from now we will assume that $\operatorname{char}(G)=p>2$.

Lemma 2.7 ([9], see also [1], Lemma 11.50 and Proposition 11.51). Let $j, k \in J$ be distinct involutions. Then

(a) $C_{G}(j k)=j J \cap k J$ is abelian and inverted by $j$;

(b) the set $\left\{C_{G}(x)^{\#} \mid x \in\left(J^{2}\right)^{\#}\right\}$ forms a partition of $\left(J^{2}\right)^{\# \text {; }}$

(c) $N_{G}\left(C_{G}(j k)\right)=C_{G}(j k) \rtimes N_{H}\left(C_{G}(j k)\right)$ is a split sharply 2-transitive group.

If $G=N H$ is split, then the group $H$ acts freely on $N$, that is for all $v \in N^{\#}$ and all $h \in H^{\#}, v^{h} \neq v$. 
Lemma 2.8 ([4], Theorem 1.1 and Corollary 1.2). Let $N$ be an abelian group, and let $H$ be a group of automorphisms of $N$. If $H$ has exponent $2^{m} \cdot 3^{n}$ for $0 \leq m$ and $0 \leq n \leq 2$ and $H$ acts freely on $N$, then $H$ is finite. Moreover, if $N H$ is a sharply 2-transitive permutation group and $n>0$, then $|N| \in\left\{5^{2}, 7^{2}, 17^{2}\right\}$ or $N$ has prime order.

Lemma 2.9. Let $N$ be an abelian group, and let $H$ be a group of automorphisms of $N$ acting freely on $N$. If $H$ is locally finite and has finite exponent, then $H$ is finite.

Proof. Denote by $\pi(H)$ the set of prime numbers that divide the order of some element of $H$. Since $H$ is locally finite, if $p \in \pi(H)$, then every Sylow $p$-subgroup of $H$ is cyclic or, if $p=2$, quaternion ([2] Theorem 10.3.1). By hypothesis $H$ has finite exponent and hence $\pi(H)$ is finite, moreover, every Sylow $p$-subgroup of $H$ is finite and hence also $H$ is finite.

\section{The $\lambda \rho$-Method}

Let $t$ be the unique involution of $H$ and fix $\vartheta \in J, \vartheta \neq t$. Since $G$ is doubly transitive, we known that $G=H \cup H \vartheta H$ ([2], Theorem 2.7.2). In particular, by the sharply 2-transitivity of $G$, for every $h \in H^{\#}$, there is a unique $\lambda(h) \in H^{\#}$ and a unique $\rho(h) \in H^{\#}$ such that

$$
\vartheta h \vartheta=\lambda(h) \vartheta \rho(h) \text {. }
$$

Thus this defines two maps $\lambda, \rho: H^{\#} \longrightarrow H^{\#}$ as in [10]. We define also

$$
\Delta(h)=\lambda(h) \rho(h) \quad \text { and } \quad \nabla(h)=\rho(h) \lambda(h)
$$

if $h \in H^{\#}$ and we extend $\Delta, \nabla$ to all $H$ putting $\Delta(1)=\nabla(1)=t$.

It is an easy matter to verify that $\rho\left(t^{-1}\right)=\lambda(t)^{-1}$; we will put $\rho(t)=u$.

By Lemma 2.5, $|\langle t \vartheta\rangle|=\operatorname{char}(G)=p>2$. By Lemma 2.7.(c) $N_{G}\left(C_{G}(t \vartheta)\right)$ is a split sharply 2transitive group with complement $N_{H}\left(C_{G}(t \vartheta)\right)$, in particular, $G$ is split if and only if $N_{H}\left(C_{G}(t \vartheta)\right)=H$. Since the subgroup $N_{H}\left(C_{G}(t \vartheta)\right)$ of $H$ assumes some importance in our arguments, then we will put

$$
\mathcal{E}_{\vartheta}(H)=N_{H}\left(C_{G}(t \vartheta)\right)
$$

in order to simplify the notation; further, if there is no loss of clarity, we simply write $\mathcal{E}(H)$ in place of $\mathcal{E}_{\vartheta}(H)$.

Lemma 3.1. Let $h \in H^{\#}$, then

$$
\lambda(\lambda(h))=\rho(\rho(h))=\Delta(\Delta(h))=h,
$$

in particular $\lambda, \rho$ and $\Delta$ are bijections form $H^{\#}$ to $H^{\#}$. Moreover,

$$
\begin{array}{ll}
\lambda(\rho(h))=\lambda(h)^{-1}, & \rho(\lambda(h))=\rho(h)^{-1}, \\
\lambda\left(h^{-1}\right)=\rho(h)^{-1}, & \rho\left(h^{-1}\right)=\lambda(h)^{-1}, \\
\Delta\left(h^{-1}\right)=\Delta(h)^{-1}, & \nabla\left(h^{-1}\right)=\nabla(h)^{-1} .
\end{array}
$$

Proof. From $\vartheta h \vartheta=\lambda(h) \vartheta \rho(h)$ we obtain $\vartheta \lambda(h) \vartheta=h \vartheta \rho(h)^{-1}$, so $\lambda(\lambda(h))=h$ and $\rho(\lambda(h))=$ $\rho(h)^{-1}$. Similarly $\rho(\rho(h))=h$ and $\lambda(\rho(h))=\lambda(h)^{-1}$.

The proof of (5) is obtained by considering the equality

$$
\rho(h)^{-1} \vartheta \lambda(h)^{-1}=(\lambda(h) \vartheta \rho(h))^{-1}=(\vartheta h \vartheta)^{-1}=\vartheta h^{-1} \vartheta
$$

and from (5) we deduce (6). 
In order to prove (3), consider

$$
\begin{aligned}
& \lambda(\Delta(h)) \vartheta \rho(\Delta(h))=\vartheta \Delta(h) \vartheta=\vartheta \lambda(h) \rho(h) \vartheta=\vartheta \lambda(h) \vartheta \vartheta \rho(h) \vartheta= \\
& h \vartheta \rho(h)^{-1} \lambda(h)^{-1} \vartheta h=h \vartheta \Delta(h)^{-1} \vartheta h=h \lambda\left(\Delta(h)^{-1}\right) \vartheta \rho\left(\Delta(h)^{-1}\right) h,
\end{aligned}
$$

so, by equating the left part of the first and the last terms of the previous equality, we obtain $\lambda(\Delta(h))=$ $h \lambda\left(\Delta(h)^{-1}\right)=h \rho(\Delta(h))^{-1}$ and hence $\Delta(\Delta(h))=\lambda(\Delta(h)) \rho(\Delta(h))=h$.

Remark B. By Lemma 3.1, we can deduce that $\langle\lambda, \rho\rangle$ is a permutation group on the set $H^{\#}$ isomorphic to $S_{3}$ and we have $\lambda(\rho(\lambda(h)))=h^{-1}=\rho(\lambda(\rho(h)))$ for every $h \in H^{\#}$ (see also Section 2 in [10]).

Lemma 3.2. The map $\nabla: H \rightarrow H$ is injective and $\nabla(h)$ is conjugate to $\Delta(h)$ for every $h \in H$. If $C$ is a conjugacy class in $H$, then $\nabla(\Delta(C)) \subseteq C$ and, if $C$ is finite, $\nabla(\Delta(C))=C$. In particular, $\widehat{Z}(H) \subseteq \nabla(H)$.

Proof. If $h \in H$, then

$$
\vartheta \nabla(h)=\vartheta \rho(h) \lambda(h)=(\lambda(h) \vartheta \rho(h))^{\lambda(h)}=(\vartheta h \vartheta)^{\lambda(h)}=h^{\vartheta \lambda(h)} .
$$

Suppose $\nabla\left(h_{1}\right)=\nabla\left(h_{2}\right)$ with $h_{1}, h_{2} \in H$, then, by (7), we can write $h_{1}^{\vartheta \lambda\left(h_{1}\right)}=h_{2}^{\vartheta \lambda\left(h_{2}\right)}$ and $h_{1}^{\vartheta \lambda\left(h_{1}\right) \lambda\left(h_{2}\right)^{-1} \vartheta}=h_{2} \in H$. Since $H$ is malnormal $\vartheta \lambda\left(h_{1}\right) \lambda\left(h_{2}\right)^{-1} \vartheta \in H$, so $\lambda\left(h_{1}\right)=\lambda\left(h_{2}\right)$ and $h_{1}=h_{2}$ by Lemma 3.1 .

Clearly, $\Delta(h)=\nabla(h)^{\lambda(h)}$, so $\Delta(h)$ and $\nabla(h)$ are conjugate. Let $C$ be a conjugacy class of $G$, then, by Lemma 3.1, $\Delta(\Delta(C))=C$ and since $\Delta(h)$ and $\nabla(h)$ are conjugate, we have $\nabla(\Delta(C)) \subseteq C$. Since $\nabla$ is injective, if $C$ is finite, then $\nabla(\Delta(C)) \subseteq C$ and this implies that $Z(H) \subseteq \nabla(H)$.

Lemma 3.3. If the map $\nabla: H \rightarrow H$ is surjective, then $G$ is split.

Proof. By Theorem 2.1, it is sufficient to prove that the unique regular element in $H \vartheta$ is $t \vartheta$. Let $h \in H \backslash$ $\{1, t\}$ and $k \in H$ with $h=\nabla(k)$. The element $h \vartheta=\rho(k) \lambda(k) \vartheta$ is conjugate to $\lambda(k) \vartheta \rho(k)=\vartheta k \vartheta \in H^{\vartheta}$, and so $h \vartheta$ fixes an element of $\Omega$.

Remark C. One can prove that $\nabla$ is surjective if and only if $G$ is split and is planar, that is, $G=N H$ and for every $h \in H^{\#}$ the map

$$
T_{h}: N \longrightarrow N \quad v \mapsto v^{-1} v^{h}
$$

is surjective (see Proposition 5.3).

There are, in each characteristic, examples of sharply 2-transitive groups that are split and in which $\nabla$ is not surjective. In the case where $H$ is periodic, it can be shown that this case can not happen (see Proposition 5.4).

Remark D. By Lemma 3.3 we deduce that if $H$ is a FC-group, then $G$ is split. This provides a more direct proof of Theorem 9.6 in [6].

The special case where $H$ is abelian has a curious history in what it has been proved at least four times. In 1952 by Tits ([13], "hidden" in the Remark 2, p. 47), in 1961 by Zemmer [16], in 1990 by Mazurov [8] and by Károlyi et al. [5].

Lemma 3.4. $\mathcal{E}(H)=\{h \in H \mid \Delta(h)=t h\}$.

Proof. We prove that $\Delta(h)=t h$ if and only if $h \in E=N_{H}\left(C_{G}(t \theta)\right)$. To do this, by Lemma 2.7 it sufficies to prove that $\left[t \vartheta,(t \vartheta)^{h}\right]=1$ for all $h \in H$. The claim is obvious if $h=1$ or $h=t$, so we assume $h \notin\{1, t\}$. Since $\vartheta t \vartheta=u^{-1} \vartheta u$ we can also write $\vartheta u^{-1} \vartheta=t \vartheta u^{-1}$ and $\vartheta u \vartheta=\vartheta t$ and hence, keeping 
in mind Lemma 3.1, if $\Delta(h)=t h$, we obtain

$$
\begin{aligned}
& t \vartheta(t \vartheta)^{h}=t \vartheta h^{-1} t \vartheta h=t\left(\vartheta h^{-1} \vartheta\right)(\vartheta t \vartheta) h=t \lambda\left(h^{-1}\right) \vartheta \rho\left(h^{-1}\right) u^{-1} \vartheta u h= \\
& t \lambda\left(h^{-1}\right)\left(\vartheta u^{-1} \vartheta\right)\left(\vartheta \rho\left(h^{-1}\right) \vartheta\right) u h=t \lambda\left(h^{-1}\right) t \vartheta u^{-1} \lambda\left(\rho\left(h^{-1}\right)\right) \vartheta \rho\left(\rho\left(h^{-1}\right)\right) u h= \\
& \lambda\left(h^{-1}\right) \vartheta \rho(h) u^{-1} \vartheta u=\lambda\left(h^{-1}\right) \vartheta \rho(h) \vartheta \vartheta u^{-1} \vartheta u=\lambda\left(h^{-1}\right) \lambda(\rho(h)) \vartheta h \vartheta t \vartheta= \\
& \rho(h)^{-1} \lambda(h)^{-1} \vartheta h \vartheta t \vartheta=\Delta\left(h^{-1}\right) \vartheta h \vartheta t \vartheta=h^{-1} t \vartheta h \vartheta t \vartheta=(t \vartheta)^{h} t \vartheta,
\end{aligned}
$$

that is $\left[t \vartheta,(t \vartheta)^{h}\right]=1$.

If $\left[t \vartheta,(t \vartheta)^{h}\right]=1$, we develop both members of $t \vartheta(t \vartheta)^{h}=(t \vartheta)^{h} t \vartheta$ obtaining

$$
t \vartheta(t \vartheta)^{h}=t \lambda\left(t h^{-1}\right) \vartheta \rho\left(t h^{-1}\right) h
$$

and

$$
(t \vartheta)^{h} t \vartheta=t h^{-1} \lambda(t h) \vartheta \rho(t h) .
$$

So $\rho\left(t h^{-1}\right) h=\rho(t h), \Delta(t h)=\lambda(t h) \rho(t h)=\rho\left(t h^{-1}\right)^{-1} \rho(t h)=h$ and finally $\Delta(h)=\Delta$ $(\Delta(t h))=t h$.

We also prove the following proposition that is not required for the proof of our theorems.

Proposition 3.5. If $u \in Z(H)$, then $G$ is split. In particular, if $\operatorname{char}(G)=3$, then $G$ is split.

Proof. Let $h$ be an element in $H^{\#}$; we have

$$
\begin{aligned}
& \lambda(t h) \vartheta \rho(t h)=\vartheta t h \vartheta=\vartheta t \vartheta \vartheta h \vartheta=u^{-1} \vartheta u \lambda(h) \vartheta \rho(h)=u^{-1} \vartheta \lambda(h) u \vartheta \rho(h)= \\
& u^{-1} \vartheta \lambda(h) \vartheta \vartheta u \vartheta \rho(h)=u^{-1} h \vartheta \rho(h)^{-1} u \vartheta t \rho(h)=h u^{-1} \vartheta u \rho(h)^{-1} \vartheta \rho(h) t= \\
& h \vartheta t \vartheta \rho(h)^{-1} \vartheta \rho(h) t=h \vartheta t h^{-1} \vartheta \lambda(h) \rho(h) t=h \lambda\left(t h^{-1}\right) \vartheta \rho\left(t h^{-1}\right) \Delta(t)
\end{aligned}
$$

and hence $\lambda(t h)=h \lambda\left(t h^{-1}\right)$, that is, $\Delta(t h)=h$ and $\Delta(h)=t h$. By Lemma 3.4 we obtain $H=\mathcal{E}(H)$ and hence $G$ is split.

If $\operatorname{char}(G)=3$, then $(\vartheta t)^{3}=1$ and $\vartheta t \vartheta=t \vartheta t$, so $u=t \in Z(H)$.

Other proofs that a sharply 2-transitive group $G$ with $\operatorname{char}(G)=3$ is split can be found in [6] (Theorem 8.7) and in [14].

The following two lemmas are a direct consequence of (7).

Lemma 3.6. Let $h$ be an element of $H^{\#}$, then $h$ and $\vartheta \nabla(h)$ have the same order.

Proof. $\vartheta \nabla(h)=\vartheta \rho(h) \lambda(h)$ is conjugate to $\lambda(h) \vartheta \rho(h)=\vartheta h \vartheta$.

Lemma 3.7. Let $w$ be an element of $H, w \neq t$. If $w \in \nabla(H)$, then $\vartheta w$ cannot be regular.

Proof. Let $h \in H$ be such that $w=\nabla(h)$. Then

$$
\vartheta w=\vartheta \rho(h) \lambda(h)=(\lambda(h) \vartheta \rho(h))^{\lambda(h)}=h^{\vartheta \lambda(h)}
$$

fixes a point of $\Omega$.

\section{Proof of Theorems 1.1 and $\mathbf{1 . 2}$}

Proof of Theorem 1.2. In $H$ there is a unique involution $t$ and hence, by Lemma 2.4, the Sylow 2-subgroups of $H$ are finite. In order to prove that $H$ is finite we just prove that $T=\left\{h \in H \mid h^{3}=1\right\}$ is 
finite. Let $h \in T^{\#}$ and $w=\nabla(h)$. By Lemma 3.6 we have $(\vartheta w)^{3}=1$, that is

$$
1=\vartheta w \vartheta w \vartheta w=\vartheta w \lambda(w) \vartheta \rho(w) w
$$

or $\vartheta=w \vartheta w \vartheta w=w \lambda(w) \vartheta \rho(w) w$ which implies $\lambda(w)=w^{-1}=\rho(w)$. Since $(\vartheta w)^{3}=1$, we have also $\left(\vartheta w^{-1}\right)^{3}=1$ and hence $\vartheta \vartheta^{w} \vartheta^{w^{2}}=w^{3}$ that is

$$
\vartheta w^{3}=\vartheta^{w} \vartheta^{w^{2}}
$$

and $\vartheta w^{3}$ should be a regular element of $G$. By hypothesis $w^{3} \in \widehat{Z}(H)$ and by Lemma $3.2 \widehat{Z}(H) \subseteq \nabla(H)$, hence, by Lemma 3.7, $\nabla\left(w^{3}\right)=1$ and $w^{3}=t$. Now $\Delta(w)=w^{-2}=w^{-3} w=t w$ and, by Lemma 3.4, $w \in \mathcal{E}(H)$. By Lemma $2.8 \mathcal{E}(H)$ is finite and hence, by Lemma 3.2, $T$ is finite. Thus $G$ is finite and the structure of $G$ is as described in Lemma 2.8 .

Proof of Theorem 1.1. Suppose $H$ is nilpotent and of exponent $2^{m} \cdot 3$ for some $m \geq 1$. Let $S$ be a Sylow 2-subgroup of $H$, since $S$ contains a unique involution, then $S$ is finite and $S \leq \widehat{Z}(H)$. Hence $H / Z(H)$ has exponent dividing 3, Theorem 1.2 applies.

As one can check (using for instance Theorem 20.7.2 in [3] and the list of exceptionals Zassenhaus' near-fields provided in [3], p. 391), if $G$ is a group that satisfies the hypotheses of the Theorem 1.1, then $H$ is necessarily cyclic. If $G=N H$ satisfies the hypotheses of the Theorem 1.2 and $N$ is not cyclic, then one of the following cases can occur:

- $N \simeq C_{5} \times C_{5}$ and $H \simeq C_{24}$, or $H \simeq C_{3} \rtimes C_{8}$, or $H \simeq S L(2,3)$;

- $N \simeq C_{7} \times C_{7}$ and $H \simeq C_{48}$, or $H \simeq C_{3} \rtimes C_{16}$, or $H \simeq G L(2,3)$;

- $N \simeq C_{17} \times C_{17}$ and $H \simeq C_{288}$, or $H \simeq C_{9} \rtimes C_{32}$.

\section{Appendix: Near-Fields and Near-Domains}

Definition II. A near-domain is a set $\mathbf{F}$ equipped with two binary operations $\oplus$ and $\odot$ such that

(II.1) $\quad(\mathbf{F}, \oplus)$ is a loop with neutral element 0 ;

(II.2) if $a \oplus b=0$, then $b \oplus a=0$;

(II.3) $\quad\left(\mathbf{F}^{\#}, \odot\right)$ is a group with neutral element 1 ;

(II.4) $0 \odot a=0$ for all $a \in \mathbf{F}$;

(II.5) $\quad a \odot(b \oplus c)=(a \odot b) \oplus(a \odot c)$ for all $a, b, c \in \mathbf{F}$;

(II.6) for every $a, b \in \mathbf{F}$ there is $\partial_{a, b} \in \mathbf{F}^{\#}$ such that

$$
a \oplus(b \oplus x)=(a \oplus b) \oplus\left(\partial_{a, b} \odot x\right)
$$

for all $x \in \mathbf{F}\left(\partial_{a, b}\right.$ is independent from $\left.x\right)$.

Definition III. A near-field is a near-domain such that $(\mathbf{F}, \oplus)$ is a group.

It is clear that a near-domain $\mathbf{F}$ is a near-field if and only if $\partial_{a, b}=1$ for every $a, b \in \mathbf{F}$.

Definition IV. A near-domain $\mathbf{F}$ is planar if for every $a, m \in \mathbf{F}$ with $m \notin\{0,1\}$ there exists an $x \in \mathbf{F}$ such that $a+m x=x$.

A planar near-domain is necessarily a near-field ([6], II.3.7 and II.5.11).

Theorem 5.1 ([6] II.6.1, II.7.1, II.7.2). Let $\mathbf{F}$ be a near-domain, then the set of one-dimensional affine transformations on $\mathbf{F}$

$$
\mathbf{T}_{2}(\mathbf{F})=\{\mathbf{F} \longrightarrow \mathbf{F} x \mapsto a \oplus m \odot x \mid a, m \in \mathbf{F}, m \neq 0\}
$$


is a group under the composition of maps. $\mathbf{T}_{2}(\mathbf{F})$ operates sharply 2-transitivity on the elements of $\mathbf{F}$ and moreover

(a) $\mathbf{T}_{2}$ (F) is split if and only if $\mathbf{F}$ is a near-field;

(b) $\mathbf{T}_{2}(\mathbf{F})$ is planar if and only if $\mathbf{F}$ is a planar near-field.

We can interpret the $\lambda \rho$-method in the language of the near-domains.

Proposition 5.2. Let $G$ be a sharply 2-transitive group on a set $\Omega$. Assume $\operatorname{char}(G) \neq 2$, let $H=G_{\alpha}$ be the stabilizer of an element $\alpha \in \Omega$ and let $t$ be the unique involution in $H$. Put $\mathbf{F}=H \dot{\cup}\{0\}$ and in $\mathbf{F}$ define two operations in the following way:

$$
\begin{gathered}
a \oplus b= \begin{cases}a \lambda\left(t a^{-1} b\right) & \text { if } a, b \in H \text { and } a \neq t b \\
0 & \text { if } a, b \in H \text { and } a=t b \\
a & \text { if } b=0 \\
b & \text { if } a=0\end{cases} \\
a \odot b= \begin{cases}a b & \text { if } a, b \in H \\
0 & \text { if } a=0 \text { or } b=0 .\end{cases}
\end{gathered}
$$

Then $(\mathbf{F}, \oplus, \odot)$ is a near-domain and $G$ is isomorphic, as permutation group, to $\mathbf{T}_{2}(\mathbf{F})$.

Moreover, if $a, b \in \mathbf{F} \backslash\{0\}=H$, then $\partial_{a, b}=a \Delta\left(t a^{-1} b\right) b^{-1}$ and hence $\mathbf{F}$ is a near-field if and only if $\Delta(h)=$ th for every $h \in H$.

Proof. A tedious but easy computation.

Proposition 5.3. Let $G$ be a sharply 2-transitive group with $\operatorname{char}(G) \neq 2$ and point stabilizer $H$ and let $\mathbf{F}$ be the associated near-domain. Then $\mathbf{F}$ is a planar near-field if and only if $H=\nabla(H)$.

Proof 1. (near-field style). Suppose $H=\nabla(H)$ and $a, m \in \mathbf{F}$ with $m \notin\{0,1\}$. If $m \neq t$ then, by hypothesis, there is $h \in H$ such that $t a^{-1} m a=\nabla(h)$ and define $x=a \lambda(h)^{-1}$. We have

$$
\begin{aligned}
a \oplus m \odot x & =a \oplus m x=a \lambda\left(t a^{-1} m x\right)=a \lambda\left(t a^{-1} m a \lambda(h)^{-1}\right) \\
& =a \lambda\left(\nabla(h) \lambda(h)^{-1}\right)=a \lambda(\rho(h))=a \lambda(h)^{-1}=x .
\end{aligned}
$$

If $m=t$ then, remembering that $\vartheta t \vartheta=u^{-1} \vartheta u$ and $\lambda(u)=u$, we define $x=a u$ and we obtain $a \oplus t \odot x=a \lambda\left(a^{-1} x\right)=a \lambda(u)=a u=x$.

Suppose F planar; by definition $\nabla(1)=t$ and $\nabla(t)=1$. Let $k \in H \backslash\{1, t\}$ and let $x \in H$ be such that $t \oplus k \odot x=x$. Then we can verify that $h=\lambda\left(t x^{-1}\right)$ is an element such that $\nabla(h)=k$.

Proof 2. (group-theoretic style). By hypothesis $\nabla(H)=H$ and hence, by Lemma 3.3, $G$ is split. So we can write $G=N H$ with $N \unlhd G$ abelian and $t$ acting by conjugation as the inversion on $N$. Let $v \in N$, since $v^{t}=v^{-1}$, there is an involution $\vartheta$ in $G$ such that $v=t \vartheta$. Fix $h \in H^{\#}$, we have to show that the map $T_{h}: N \longrightarrow N, y \mapsto y^{-1} y^{h}$ is surjective. If $h=t$, the claim is trivial and hence we suppose $h \notin\{1, t\}$. Our assertion is proved if we can find an element $k \in H$ such that $\left(t \vartheta^{k}\right)^{-1}\left(t \vartheta^{k}\right)^{h}=t \vartheta$. If we choose $k$ such that $h=t \nabla(\lambda(t k))^{-1}$, then, remembering that, since $G$ is split, is $\Delta(k)=t k$, we obtain

$$
\begin{aligned}
& \left(t \vartheta^{k}\right)^{-1}\left(t \vartheta^{k}\right)^{h}=k^{-1} \vartheta k h^{-1} k^{-1} \vartheta k h=k^{-1} \vartheta k t \nabla(\lambda(t k)) k^{-1} \vartheta k t \nabla(\lambda(t k))^{-1}= \\
& k^{-1} \vartheta t k \rho(\lambda(t k)) \rho(\rho(t k)) k^{-1} \vartheta t k(\rho(\lambda(t k)) \rho(\rho(t k)))^{-1}=k^{-1} \vartheta k \rho(t k)^{-1} \vartheta \rho(t k)= \\
& k^{-1} \vartheta(\lambda(t k) \vartheta \rho(t k))=k^{-1} \vartheta(\vartheta t k \vartheta)=t \vartheta,
\end{aligned}
$$

and the proof is complete. 
Remark E. The two proofs of Proposition 5.3 show that the definitions given in Remark C and Definition IV are actually equivalent.

Remark F. If $\operatorname{char}(G)=2$, then we can choose and involution $\vartheta \in G$ and, if $H=G_{\alpha}$, we can define as above the maps $\lambda, \rho, \Delta$ and $\nabla$. In this case, if we put $t=1$, then it is not difficult to verify that Propositions 5.2 and 5.3 are still true. Moreover, $G$ is split if and only if $H=\mathcal{E}(H)=\{h \in H \mid \Delta(h)=h\}$.

We conclude this short appendix with the following result.

Proposition 5.4. Let $G$ be a split sharply 2-transitive group. If the point stabilizer $H$ is periodic, then $G$ is planar.

Proof. Write $G=N H$ with $N \unlhd G$ and $N \cap H=\{1\}$. Since $H$ is periodic, then, by Remark A, $\operatorname{char}(G)=$ $p>0$ and hence $N$ is an elementary abelian $p$-group acted freely by $H$. Let $h \in H^{\#}$ be an element of order $\ell$, then $(p, \ell)=1$ and hence there is a positive integer $\delta$ such that $p^{\delta} \equiv 1 \bmod \ell$. Let $\bar{h}$ be the automorphism induced by conjugation by $h$ on $N$ and let $q=p^{\delta}$. In the $\operatorname{ring} \operatorname{End}(N)$, we have

$$
\left(T_{h}\right)^{q}=(-1+\bar{h})^{q}=-1+\bar{h}^{q}=-1+\bar{h}=T_{h}
$$

that is, $T_{h}^{q-1}=\mathrm{id}_{N}$, and hence $T_{h}$ is a bijection.

\section{ORCID}

Enrico Jabara (D) http://orcid.org/0000-0001-5913-1820

\section{References}

[1] Borovik, A., Nesin, A. (1994). Groups of Finite Morley Rank. Oxford Logic Guides, Vol. 26. New York: Oxford Science Publications, The Clarendon Press, Oxford University Press.

[2] Gorenstein, D. (1980). Finite Groups, 2nd ed. New York: Chelsea Publishing Co.

[3] Hall, M. (1976). The Theory of Groups, Reprinting of the 1968 edition. New York: Chelsea Publishing Co.

[4] Jabara, E., Mayr, P. (2009). Frobenius complements of exponent dividing $2^{m}$. 9. Forum Math. 21(2):217-220.

[5] Károlyi, Gy., Kovács, S. J., Pálfy, P. P. (1990). Doubly transitive permutation groups with abelian stabilizers. Aequationes Math. 39(2-3):161-166.

[6] Kerby, W. (1974). On Infinite Sharply Multiply Transitive Groups. Hamburger Mathematische Einzelschriften, Neue Folge, Heft 6. Göttingen: Vandenhoeck \& Ruprecht.

[7] Mayr, P. (2006). Sharply 2-transitive groups with point stabilizer of exponent 3 or 6. Proc. Am. Math. Soc. 134(1):9-13.

[8] Mazurov, V. D. (1990). Doubly transitive permutation groups. Sibirsk. Mat. Zh. 31(4):102-104, 222; English translation in Siberian Math. J. 31(4):615-617.

[9] Nesin, A. (1992). Notes on sharply 2-transitive permutation groups. Doga Mat. 16(1):69-84.

[10] Peterfalvi, T. (2005). Existence d'un sous-groupe normal régulier dans certains groupes 2-transitifs. J. Algebra 294(2):478-488.

[11] Rips, E., Segev, Y., Tent, K. A sharply 2-transitive group without a non-trivial abelian normal subgroup. arXiv: 1406.0382

[12] Suchkov, N. M. (2001). On the finiteness of some exactly doubly transitive groups. Algebra Logika 40(3):344-351, 374; English translation in Algebra Logic 40(3):190-193.

[13] Tits, J. (1952). Généralisations des groupes projectifs basées sur leurs propriétés de transitivité. Acad. Roy. Belgique. Cl. Sci. Mm. Coll. in Facs. 2, Brussels.

[14] Türkelli, S. (2004). Splitting of sharply 2-transitive groups of characteristic 3. Turkish J. Math. 28(3):295-298.

[15] Zassenhaus, H. (1935). Über endliche Fastkörper. Abh. Math. Semin. Hamb. Univ. 11:187-220.

[16] Zemmer, J. L. (1961). On a class of doubly transitive groups. Proc. Am. Math. Soc. 12:644-650. 